\title{
Factors Inducing Literature Anxiety for Students Studying Literature in English
}

\author{
Agnes Wei Lin Liau* \\ Universiti Sains Malaysia, Penang, Malaysia \\ https://orcid.org/0000-0001-5836-5270 \\ George Boon Sai Teoh \\ Universiti Sains Malaysia, Penang, Malaysia \\ https://orcid.org/0000-0003-2071-1849
}

\begin{abstract}
Students have been plagued with various types of anxieties in the educational context, hence we propose to introduce the concept of literature anxiety in this study. This study investigates the possible factors inducing literature anxiety from the perspectives of university students studying Literature in English at tertiary level, and records their emotional experiences during their learning encounters with Literature in English. The study also presents how these students make sense of their Literature in English learning and how their knowledge and perceptions of Literature in English have influenced or affected their experience of literature anxiety. SPSS analysis of the data procured through the Literature Anxiety Scale (LITAS) provided evidence concerning students' literature anxiety and the factors they perceive inducing literature anxiety for them. The literature anxiety-inducing factors were identified as situational, dispositional, and environmental factors. The findings obtained showed that students' literature anxiety does debilitate their studying of Literature in English. The implications are that educators should teach more attentively and be sensitized to learners' possible experience of anxiety.
\end{abstract}

Keywords: academic achievement; emotional experiences; literature anxiety; Literature in English; literature learning

\section{Introduction}

Spielberger and Vagg (1995) described fear and anxiety as being "clearly recognized as significant aspects of human behavior in ancient Egypt, the Old Testament, in Greek and Roman Literature" (p. 3). Indisputably, anxiety is and has been a concern for millennia. Lately, there has been an upsurge in research on anxiety and its effects on academic achievement, augmenting research in

*Corresponding author: Agnes Wei Lin Liau; Email: agnes@usm.my 
various aspects of anxiety. Anxiety impacts many contexts, not sparing educational contexts (Deieso \& Fraser, 2018; Zaccoletti et al., 2020). Issues surrounding anxiety and anxiety disorders are global concerns (Luttenberger et al., 2018). Anxiety disorders have been recorded as the most common mental health disorders suffered across societies and linked with notable negative consequences for humans (Reardon et al., 2018). Some studies have documented that anxiety could increase paranoid ideation (Salza et al., 2020). Anxiety disorders have effects across age groups. A study by Martinussen et al. (2019) found that older adults suffering from depression and a comorbid anxiety disorder showed more deterioration in their memory during a four-year period in comparison to older adults without a comorbid anxiety disorder. These facts stress the necessity for research on anxiety.

MacIntyre and Gardner (1991, as cited in Ellis, 1994) stated that anxiety can be hypothesized to influence three stages of the learning process - the input, processing, and output stages. However, most research has analyzed the output stage and there has not been much research on the input and processing stages. In the study of anxiety, the measurement of anxiety should also be stressed. This has led to the development of a myriad anxiety scales, which have all been scrutinized and critiqued. Many studies have examined the role of anxiety in second language learning. Many researchers (MacIntyre, 1999, as cited in Cheng, 2004) have recommended conceptualizing second or foreign language anxiety as a unique anxiety specific to the second language learning context. Research on second language learning has emphasized the impact of anxiety on language learning (Cheng, 2017; Huang, 2018). Foreign language anxiety has also been studied in association with socio-biographical factors (Jiang \& Dewaele, 2020). Aydin (2016) posited that foreign language teaching anxiety needs to be addressed too since certain teachers face anxiety in their foreign language teaching activities. In the foreign language learning classroom, Horowitz et al. (1986) stipulated that the consequences of anxiety can resonate outside the classroom. Language anxiety has also been researched via the perspectives of language teachers, where experienced teachers provided their accounts of how they perceived language anxiety (Ohata, 2005).

Classroom learners react to the learning situations in which they are placed in various affective ways which can impact their self-perceptions and induce negative emotions (Ellis, 1994; Lou \& Noels, 2020). MacIntyre, (1995) stated that there has been a marked growth in research into the anxieties of everyday life over the past 20 years. Increasing focus is evident regarding research on anxiety concerning a range of other education-related areas, including statistics anxiety, computer anxiety, and library anxiety. In addition, other types of anxiety-inducing experiences exist in the field of education, including anxiety related to tests, mathematics, and foreign languages. These anxiety experiences hamper students' ability to effectively undertake their tasks in their learning contexts. Anxiety related to sitting for a test is evidence of test anxiety (Putwain et al., 2010). However, we have not found any research to date on literature anxiety and hence this study addresses this research gap in the types of anxiety experienced in educational settings. 


\section{Literature Anxiety}

Endler and Kocovski (2001) enumerated that anxiety has been defined as "a trait, a state, a stimulus, a response, a drive and as a motive" (p. 232). However, there are various interpretations of the word anxiety, and it is difficult to pinpoint a universally agreed upon definition of anxiety. Indefiniteness surrounds the term anxiety as it can be an ambiguous construct and has received numerous conceptualizations. However, a common thread running through the definitions would be the fact that anxiety can be an impediment to the individual if not checked. We define literature anxiety as referring to the anxiety that students encounter when studying literature, undertaking literary analyses, or undergoing a literature course. Literature anxiety subscribes generally to an anxiety reaction to situations where students of literature are confronted with various forms of literature learning as well as teaching.

It is imperative that we study how anxiety can affect human lives and why individuals succumb to anxiety. Therefore, this study intends to extend scholarship on anxiety experienced among students in a learning context by offering insights into the literature anxiety experiences of students studying Literature in English at a local university. This study further enriches as well as sensitizes our understanding of literature anxiety as seen and felt through the eyes and the words of these students. This study features the factors inducing literature anxiety for these students studying Literature in English at tertiary level, who recorded experiencing literature anxiety at high, medium, and low levels, respectively. Studying Literature in English is essential for these students who are the participants for this study. In their study of English literature, they are required to read and comprehend the prescribed texts, think critically, and make informed decisions regarding their interpretation and analysis of the literary texts studied. For literature-anxious students/individuals, the interpretation and comprehension of literary texts could represent a psychological stress that is interpreted as threatening their self-esteem. If accumulated failure in interpreting or comprehending literary texts is experienced, this may lead the student or individual to view these situations as ego-threatening and a self-perpetuating cycle is formed.

In attempting to code the factors inducing students' experiences of literature anxiety, we refer to Baloğlu's (2003) categorization of the three main factors that induce statistics anxiety. Baloğlu (2003) stated in his research on statistics anxiety among college students that the "causes" of statistics anxiety are normally categorized around three main factors - dispositional, situational, and environmental. We applied the three categorizations to designate the factors inducing the participants' literature anxiety. Baloğlu (2003) explained that dispositional factors include psychological as well as emotional characteristics that would refer to attitudes towards statistics, perceptions, and self-concept. Situational factors would refer to "immediate factors that result from statistics courses and include, but are not limited to, statistics teachers, nature of statistics courses ... and statistics terminology" (Baloğlu, 2003, p. 856). Environmental factors refer to factors that have previously influenced the student or individual before entering the statistics course (Baloğlu, 2003). As examples, this would 
relate to a person's age, gender, ethnicity, previous background in mathematics, as well as educational level.

\section{Methodology}

\subsection{Sample}

We selected 21 participants (13 females and 8 males) based on an initial survey which was run to obtain students' literature anxiety scores. The participants were accordingly selected based on their scores of high, medium, and low anxiety.

\subsection{Procedure}

The research procedures for this study are illustrated in Figure 1. We first conducted a survey, which was completed by 101 participants. From these participants, 21 were selected for this study based on their literature anxiety scores. The participants completed the Literature Anxiety Scale (LITAS). The scale comprised 48 items which assessed the literature anxiety of the participants. The internal consistency of the LITAS was calculated to be 0.94 . This score should not be below 0.8 , for this means that repeated administrations will cover less than 64 per cent of the same ground and that the error component will be more than one-third (Oppenheim, 1992). A good level of internal consistency was therefore achieved by the scale. Next, the 21 participants were interviewed, with each interview lasting about an hour.

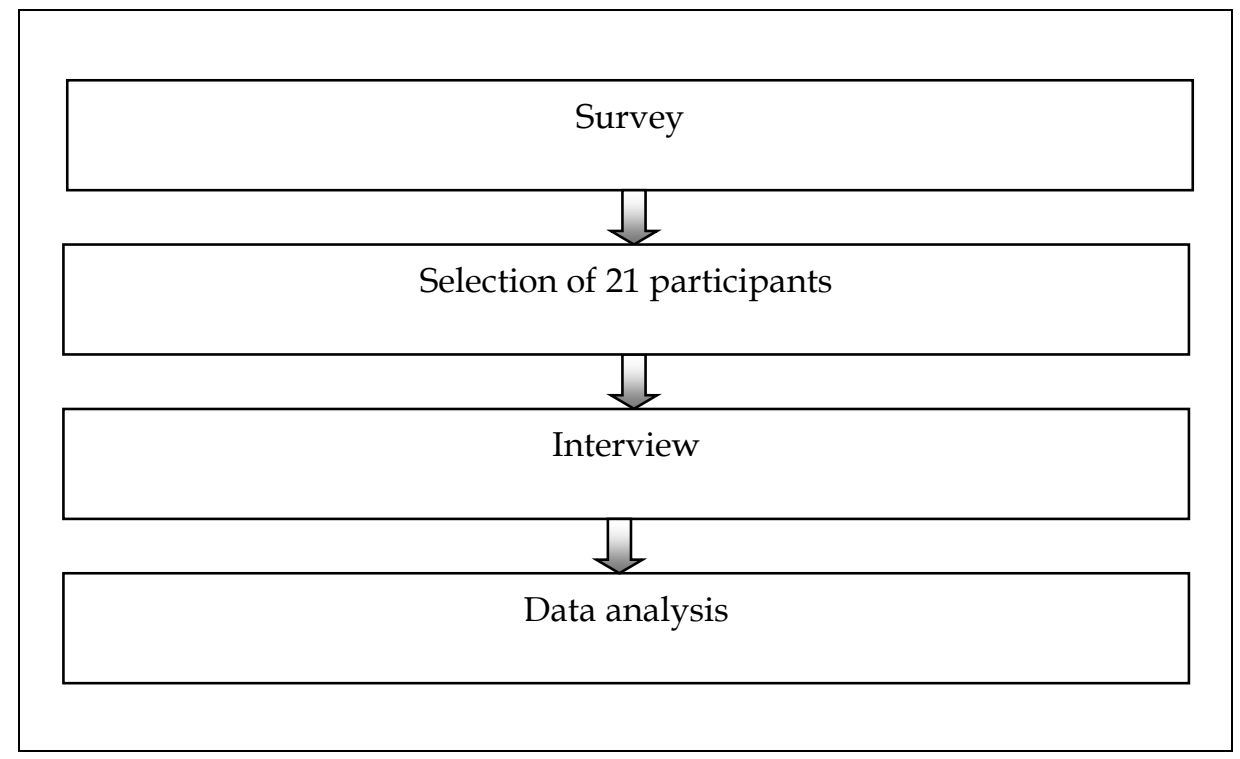

Figure 1: Research procedures

\subsection{Coding}

Participants were categorized in high-anxiety, medium-anxiety, and low-anxiety groups according to their literature anxiety scores which were obtained from the data generated by the survey. We employed Grounded Theory (Corbin \& Strauss, 2008) and content analysis (Bryman, 2004) to analyze the qualitative data collected in this phase of the study. Under the Grounded Theory approach, we first started with the open coding stage and then proceeded to the axial 
coding stage. In the open coding stage, we located possible themes and provided initial codes to begin condensing the interview data into categories (Neuman, 2000). In the axial coding stage, as Neuman (2000) termed it, we made a "second pass" through the data. In comparison to the open coding stage, the axial coding stage required starting the process of coding with a planned and organized group of initial codes. We placed more emphasis on the codes to review and examine the codes effectively to achieve a coherent order. First, we transcribed the recorded interviews with the participants. We then applied an open coding process on the resulting data and coded the data with codes representing terms that would encompass as near as possible those words uttered by the participants in the interviews.

For example, participants used the words "anxious" and "ambiguous" to allude to the "subjectivity of interpretation" they faced, as it was this aspect of studying Literature in English that especially made them experience literature anxiety. The subjectivity of interpretation and the need to provide the "correct" response often gave rise to literature anxiety among these participants. In addition, participants used the words "worried", "tension", and "boring" to allude to the "literariness of language" in Literature in English study. This element of Literature in English also caused the participants to experience literature anxiety. Finally, participants used the words "hate", "weak", and "hard" to allude to their English language level and competence. Some participants lamented their poor and inadequate English proficiency level as this made it difficult for them to understand some of the course content. They thus felt that they had to struggle through their Literature in English courses, and this induced literature anxiety for them.

The situational factors that the participants reported causing them to experience literature anxiety were premised upon the factors that arose externally from the program. These include the characteristics of Literature in English as a subject being taught, the course structure itself, the lecturers teaching the Literature in English courses, the nature of the tutorial groups, the time issue in the program, and lastly, their comprehension of the Literature in English course content. The dispositional factors that the participants reported causing them to experience literature anxiety were based on internal factors, which include their perceptions of their attitudes towards Literature in English and their ability to undertake literary tasks. Lastly, the environmental factors that the participants reported causing them to experience literature anxiety were related to what they had "brought" with them upon entering the program, such as their educational background and their age.

Furthermore, the later operational definition differed from the earlier operational definition. We refined the meaning of inadequacy of time to include not just the insufficiency of time per se, but also the sense of how time is divided for students per semester in terms of the number of subjects they have to pursue. The participants reported that they had to juggle their time between the Literature in English subjects, Linguistics subjects, as well as their minor subjects, as they had to major in Linguistics as well as Literature in English. 
Since most of them considered Literature in English challenging, they felt obliged to allocate more of their time to this course. However, the division of time did not often allow them to do just that, since they had to also concentrate on their other Linguistics subjects and their respective minor subjects which they had to take to fulfil the criteria for graduation.

Figure 2 depicts the categorization of the coding of factors inducing literature anxiety for the participants.

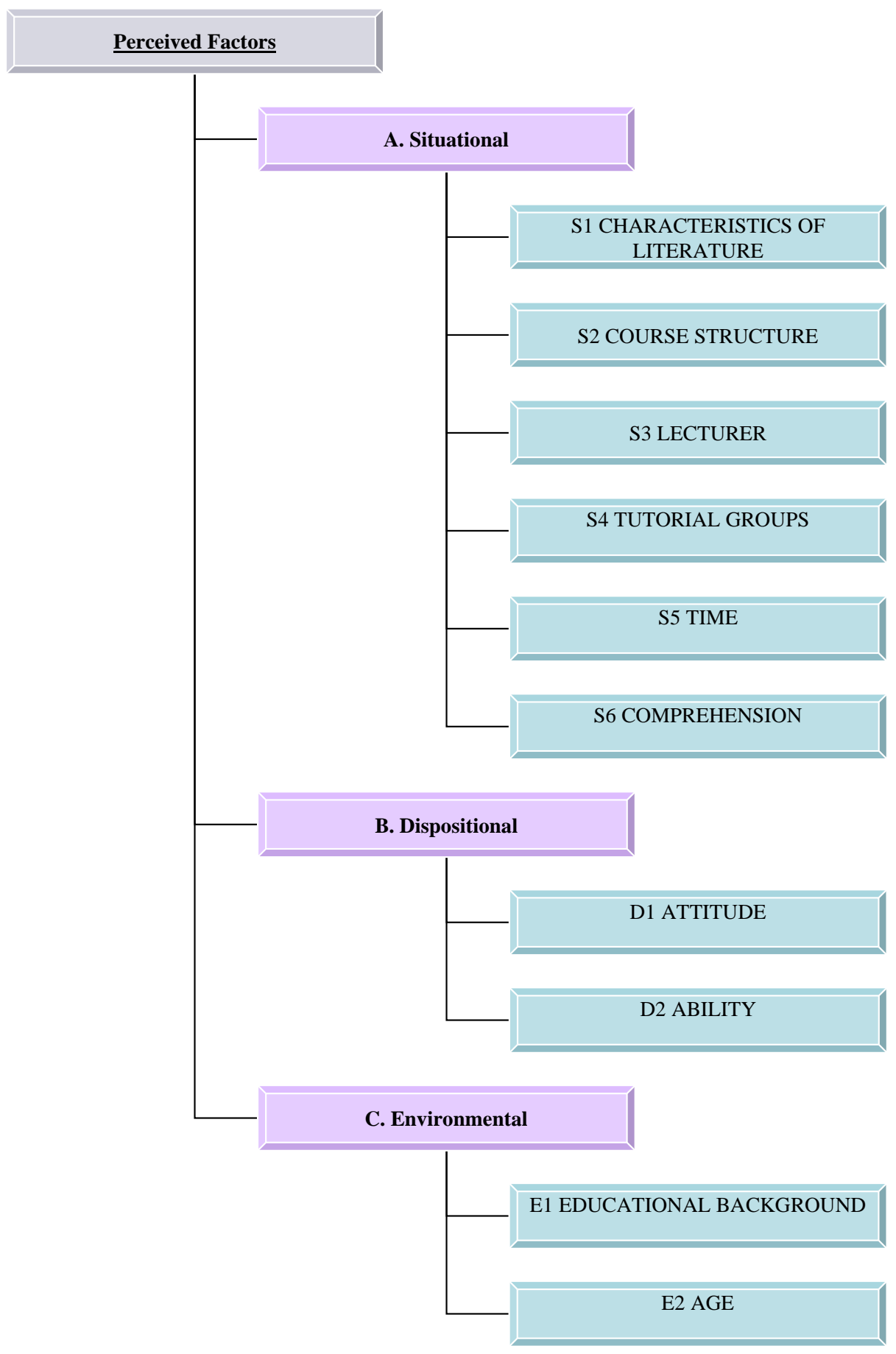

Figure 2: Perceived factors reported inducing literature anxiety 
As shown in Figure 2, participants indicated three main categories of factors, namely situational, dispositional, and environmental. Within each of these main categories, codes were identified. For the situational factor, these were S1 (characteristics of literature), S2 (course structure), S3 (lecturer), S4 (tutorial groups), S5 (time), and S6 (comprehension). The codes for the dispositional factor were D1 (attitude) and D2 (ability). Finally, the codes for the environmental factor were E1 (educational background) and E2 (age).

\section{Findings and Discussion}

Table 1 portrays the codes, the operational definitions, and the examples illustrating these codes that were refined until saturation in an iterative manner. The factors delineated in Table 1 are based on Baloğlu's (2003) categorization of the factors causing statistics anxiety. These are the situational, dispositional, and environmental factors. The factors and their codes, as mentioned above, are represented. Table 1 presents operational definitions of the codes as well as some sample excerpts from the interviews with the participants.

Table 1: Codes, operational definitions, and examples delineating situational, dispositional, and environmental factors

\begin{tabular}{lll}
\hline Code & Operational definition & Examples \\
\hline S1 & Utterances depicting that & Literature is very subjective. Sometimes even \\
(characteristics & participants experienced & when we are in class, we are giving our ideas, we \\
& literature anxiety due to & feel that we understand what we have read, what \\
& certain characteristics of & we analyzed, and what we think we derived from \\
& literature, such as & the text is okay, but when we put it forth to our \\
& English language level of lecturers, they say, "No, it's actually this way." \\
& texts, subjectivity of & So, you feel that you are wrong. Ah, that makes \\
& interpretation, and & me anxious. We know we understand the text in \\
& literary language. & such a way, and we can see it from that way, but \\
& & why is it not accepted?
\end{tabular}

Hard to understand, very ambiguous. If in one page I don't understand, it is boring to continue.

S2 (course structure)

S3 (lecturer)
Utterances depicting that the nature of the Literature in English courses or the requirements of the courses that have to be met by the students are perceived as posing anxiety for the participants.
It is getting heavier, the depth we go into. Tough.

Literature is more than just reading a book. It is about how you feel about the book, what you feel about it. When I first started taking literature, I didn't know it was going to be this tough. When I started in the first semester it was about new things. The second and third semesters, getting difficult. The more you get to know about it, the more strange. The moment that we know we have to study all these things, I get anxious.

Sometimes some lecturers cannot take it when we throw back a question at them. They feel we're questioning their authority, you know, like me being a senior student in here, sometimes the lecturer's age and mine would be around the same, you know. So, when we question them, 


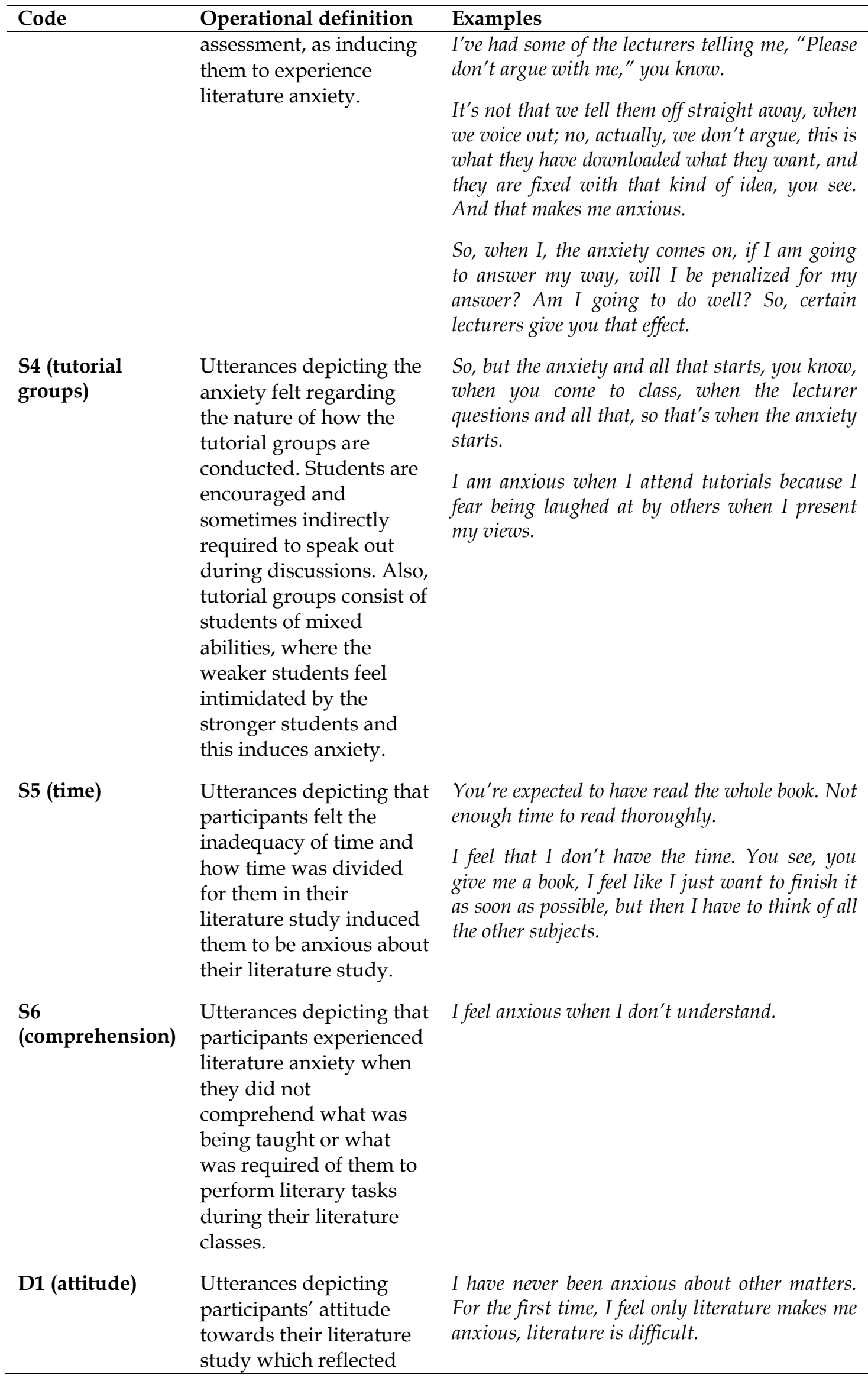




\begin{tabular}{|c|c|c|}
\hline Code & Operational definition & Examples \\
\hline & $\begin{array}{l}\text { their experience of } \\
\text { literature anxiety. }\end{array}$ & $\begin{array}{l}\text { I hate literature, this subject, because it is } \\
\text { difficult to get the double meaning. I hate the } \\
\text { lecturer too! }\end{array}$ \\
\hline \multirow[t]{2}{*}{ D2 (ability) } & \multirow{2}{*}{$\begin{array}{l}\text { Utterances depicting } \\
\text { participants' feelings } \\
\text { that they lacked the } \\
\text { ability to undertake } \\
\text { literary tasks, } \\
\text { understand literature, } \\
\text { and generally to study } \\
\text { literature, which } \\
\text { induced the experience } \\
\text { of literature anxiety. }\end{array}$} & $\begin{array}{l}\text { Literature is very hard, I always write nonsense, } \\
\text { no connection, too long, difficult to pass. I } \\
\text { understand the lecture, I don't understand what } \\
\text { I am writing, don't know how to apply the } \\
\text { theory. }\end{array}$ \\
\hline & & $\begin{array}{l}\text { There are poems where the words are not clear; } \\
\text { sometimes there are words that I have never seen } \\
\text { before. }\end{array}$ \\
\hline \multirow[t]{2}{*}{$\begin{array}{l}\text { E1 (educational } \\
\text { background) }\end{array}$} & \multirow{2}{*}{$\begin{array}{l}\text { Utterances depicting that } \\
\text { participants felt their } \\
\text { educational background } \\
\text { has affected them to } \\
\text { experience literature } \\
\text { anxiety. }\end{array}$} & $\begin{array}{l}\text { When I entered the class, it was very difficult for } \\
\text { me. My basic is not good. }\end{array}$ \\
\hline & & $\begin{array}{l}\text { When I read, the whole page is full of } \\
\text { translations, English into Malay. I understand } \\
\text { in Malay. I think that I am really weak in } \\
\text { English. Actually, I am much better in } \\
\text { Mandarin. I went to a Chinese primary school. }\end{array}$ \\
\hline E2 (age) & $\begin{array}{l}\text { Utterances depicting that } \\
\text { participants felt their age } \\
\text { has affected them to } \\
\text { experience literature } \\
\text { anxiety. }\end{array}$ & $\begin{array}{l}\text { When I was in school, I liked English; the } \\
\text { younger I was the less anxious I was. }\end{array}$ \\
\hline
\end{tabular}

The frequencies for the codes appearing in the interviews were counted (presented in Table 2 and discussed later). The participants were divided according to their literature anxiety groups, namely high, medium, and low. Based on the frequencies of the situational, dispositional, and environmental factors reported, the following findings were recorded.

The high-anxiety group had the highest frequency count for these factors:

1. S4 (tutorial groups, 9 statements);

2. S6 (comprehension, 24 statements); and

3. E1 (educational background, 12 statements).

The medium-anxiety group had the highest frequency count for these factors:

1. S1 (characteristics of literature, 30 statements);

2. S2 (course structure, 18 statements);

3. D2 (ability, 25 statements); and

4. E2 (age, 4 statements).

Lastly, the low-anxiety group had the highest frequency count for this factor:

1. D1 (attitude, 16 statements).

Regarding the situational factors, the most mentioned situational factor across the three anxiety groups was S3 (lecturer), with 77 statements, and the least 
mentioned situational factor was S5 (time), with only 6 statements. The most mentioned dispositional factor was D2 (ability), with 55 statements across the three anxiety groups, and the most mentioned environmental factor was E1 (educational background), with 15 statements across the three anxiety groups. Among the three overall categories of factors, situational factors were mentioned most frequently, with a total of 231 statements. Environmental factors were mentioned least frequently, with a total of 22 statements.

As depicted in Table 1, the operational definition for each code reflects the utterances of the participants which captured the essence of what they described about their experience of literature anxiety. The high-anxiety group recorded the highest frequency count for S6 (comprehension), which referred to experiences of anxiety when they did not understand what was taught or what they were required to undertake in their literature classes. An example excerpt from one of the high-anxiety participants attributing their anxiety to non-comprehension is shown here:

"I feel anxious when I don't understand."

Hence, comprehension was a major factor in making these participants experience literature anxiety. As for the medium-anxiety group, the code recording the highest frequency count was S1 (characteristics of literature). Here, participants explained that their anxiety was caused by certain characteristics of literature, such as the English language level found in their prescribed texts, the subjectivity of interpretation, and the literary language contained in their respective texts which hindered their understanding. This is reflected in the following interview excerpts:

"Literature is very subjective. Sometimes even when we are in class, we are giving our ideas, we feel that we understand what we have read, what we analyzed, and what we think we derived from the text is okay, but when we put it forth to our lecturers, they say, "No, it's actually this way." So, you feel that you are wrong. Ah, that makes me anxious. We know we understand the text in such a way, and we can see it from that way, but why is it not accepted?"

"Hard to understand, very ambiguous. If in one page I don't understand, it is boring to continue."

Hence, as reflected in these participants' words, they were affected by aspects of literature that oversaw technical characteristics. These included the level of the English language contained in certain literary texts. It also included the subjective nature of interpreting literary texts that can cause students confusion as to which is the right answer to the questions asked. Lastly, it involved the literariness of the language in literary texts. The feelings encapsulated within these excerpts suggest the technical aspects of literature inhibited successful learning on the part of the participants and hence induced anxiety to develop within them.

As for the low-anxiety group, these participants were most affected by the dispositional factor D1 (attitude). This code refers to students' own attitude 
towards their literature learning impacting their experience of literature anxiety. The way in which these participants viewed studying literature led them to hold such attitudes and is exemplified in the below excerpts:

"I have never been anxious about other matters. For the first time, I feel only literature makes me anxious; literature is difficult.

I hate literature, this subject, because it is difficult to get the double meaning. I hate the lecturer too!"

As presented, these participants mainly experienced literature anxiety induced by various factors under the situational and dispositional factors. Interestingly, when taken across the three groups of participants of high, medium, and low anxiety, the highest frequency count for the most mentioned situational factor inducing literature anxiety was for S3 (lecturer). The following two excerpts depict that certain aspects of lecturers' behavior can become anxiety-provoking elements for students. Certain behavioral patterns perhaps exhibited by the lecturers are deemed as not conducive to promoting literature learning and hence subsequently mar the learning process of the students in addition to causing them literature anxiety.

"Sometimes some lecturers cannot take it when we throw back a question at them. They feel we're questioning their authority, you know, like me being a senior student in here, sometimes the lecturer's age and mine would be around the same, you know. So, when we question them, I've had some of the lecturers telling me, "Please don't argue with me," you know. It's not that we tell them off straight away, when we voice out; no, actually, we don't argue, this is what they have downloaded what they want, and they are fixed with that kind of idea, you see. And that makes me anxious."

"So, when I, the anxiety comes on, if I am going to answer my way, will I be penalized for my answer? Am I going to do well? So, certain lecturers give you that effect."

Under the dispositional codes, D2 (ability) was the most frequently mentioned dispositional factor across the three groups of participants. The following two examples of participant accounts exemplify how they perceived themselves as unable to perform well in literature due to the lack of knowledge and ability on their part. This lack of knowledge on theory application hindered their comprehension of the subject.

"Literature is very hard, I always write nonsense, no connection, too long, difficult to pass. I understand the lecture, I don't understand what I am writing, don't know how to apply the theory."

"There are poems where the words are not clear; sometimes there are words that I have never seen before."

E1 (educational background) was the most mentioned environmental factor across the three groups of participants. The following example excerpts record how these two participants attributed their poor performance due to their 
educational backgrounds prior to entering the course program. They viewed themselves as being weak in terms of background knowledge and language proficiency, which in turn made them anxious in the literature class.

"When I entered the class, it was very difficult for me. My basic is not good."

"When I read, the whole page is full of translations - English into Malay. I understand in Malay. I think that I am really weak in English. Actually, I am much better in Mandarin. I went to a Chinese primary school."

This study has provided valuable insights into the possible factors inducing literature anxiety as experienced by the participants. Firstly, the possible factors participants perceived as inducing literature anxiety for them have been categorized under three main categories, namely situational, dispositional, and environmental factors. These were all divided into various codes, as explained earlier.

Based on the findings of the frequency counts for the perceived situational, dispositional, and environmental factors, the high-anxiety group had the highest frequency counts for factors S4 (tutorial groups), S6 (comprehension), and E1 (educational background). Among these three factors, the factor S6 (comprehension) had the highest frequency count, with 24 statements.

The medium-anxiety group had the highest frequency counts for factors S1 (characteristics of literature), S2 (course structure), D2 (ability), and E2 (age). Among these factors, the factor S1 (characteristics of literature) had the highest number of frequency counts. The medium-anxiety group mentioned this factor most as compared to the other factors. The low-anxiety group had the highest frequency counts for the dispositional factor D1 (attitude) among the situational, dispositional, and environmental factors.

The high-anxiety group mentioned the S4 (tutorial groups) factor most compared to the other two anxiety groups. The high-anxiety group also mentioned the $\mathrm{S} 6$ (comprehension) factor most compared to the medium-anxiety group, who in turn mentioned this factor more than the low-anxiety group. Furthermore, the high-anxiety group mentioned the E1 (educational background) factor more in comparison to the other two anxiety groups. Thus, the results indicate that the high-anxiety group of participants tended more to attribute their literature anxiety to factors requiring some form of evaluation of their performance. For example, in tutorial groups, they are anxious about their answers to questions posed or about having to contribute verbally and then experience rejection. In interpreting literary texts, they are anxious because they find it difficult to understand the texts. As for educational background, some of them felt that their educational background had not prepared them suitably for this course. Among the factors, the situational factor most frequently reported to induce literature anxiety among the participants was the S3 (lecturer) factor. Most of the participants who experienced literature anxiety mentioned this factor as inducing their literature anxiety. Thus, literature anxiety could be said 
to be mainly due to the lecturer or certain lecturers' style of teaching and assessment of the literature courses. Instead of facilitating Literature in English learning, this served to "facilitate" literature anxiety among these participants.

Table 2 presents the comparison of the individual situational, dispositional, and environmental factors using Friedman's test. The situational factor S3 (lecturer) had the highest mean rank (7.31). The second highest mean rank (7.05) was for the situational factor S6 (comprehension), and the third highest was for the situational factor S1 (characteristics of literature), with a mean rank of 7.02. Fourth was the dispositional factor D2 (ability), with a mean rank of 6.50, and fifth was the dispositional factor D1 (attitude), with a mean rank of 5.79. Sixth was the situational factor S2 (course structure, 5.62), seventh the situational factor S4 (tutorial groups, 4.10), and eighth the environmental factor E1 (educational background, 4.05). Ninth was the environmental factor E2 (age), with a mean rank of 3.93, which leaves the situational factor S5 (time) with the lowest mean rank of 3.64 .

Table 2: Comparison of situational, dispositional and environmental factors using Friedman's test

\begin{tabular}{ll}
\hline Code & Mean rank \\
\cline { 2 - 3 } S1 & 7.02 \\
S2 & 5.62 \\
S3 & 7.31 \\
S4 & 4.10 \\
S5 & 3.64 \\
S6 & 7.05 \\
D1 & 5.79 \\
D2 & 6.50 \\
E1 & 4.05 \\
E2 & 3.93 \\
\hline
\end{tabular}

$* * * p<0.000$

Table 3 presents the results of the multiple comparisons of the situational, dispositional, and environmental factors for the participants using the Wilcoxon signed-rank test. The multiple comparisons were conducted after a significant difference was seen in the results of the Friedman test comparing the individual situational, dispositional, and environmental factors. 
Table 3: Multiple comparisons of the situational, dispositional, and environmental factors for the participants using the Wilcoxon signed-rank test

\begin{tabular}{|c|c|c|c|c|c|c|c|c|c|c|}
\hline & S1 & S2 & S3 & S4 & S5 & S6 & D1 & D2 & E1 & E2 \\
\hline $\mathrm{S} 1$ & - & & & & & & & & & \\
\hline $\mathrm{S} 2$ & NS & - & & & & & & & & \\
\hline $\mathrm{S} 3$ & NS & $0.032 *$ & - & & & & & & & \\
\hline $\mathrm{S} 4$ & $0.005^{* *}$ & NS & $0.003 * *$ & - & & & & & & \\
\hline S5 & $0.002 * *$ & $0.005^{* *}$ & $0.001 * * *$ & NS & - & & & & & \\
\hline S6 & NS & $0.048 *$ & NS & $0.001 * * *$ & $0.001 * * *$ & - & & & & \\
\hline D1 & NS & NS & $0.03 *$ & $0.031 *$ & $0.005 * *$ & NS & - & & & \\
\hline D2 & NS & NS & NS & $0.019 *$ & $0.003 * *$ & NS & NS & - & & \\
\hline E1 & $0.009 * *$ & NS & $0.002 * *$ & NS & NS & $0.001 * * *$ & NS & $0.004 * *$ & - & \\
\hline E2 & $0.002 * *$ & $0.01 * *$ & $0.000 * * *$ & NS & NS & $0.001 * * *$ & $0.007 * *$ & $0.004 * *$ & NS & - \\
\hline
\end{tabular}

$* \mathrm{p}<0.05, * * \mathrm{p}<0.01, * * * \mathrm{p}<0.001$

The results in Table 3 denote that there was a significant difference between S1 (characteristics of literature, mean rank 7.02) and the following factors:

- S4 (tutorial groups, mean rank 4.10), with $p=0.005^{* *}$; and

- S5 (time, mean rank 3.64), with $p=0.002^{* *}$.

This suggests that the participants were more inclined to view the situational factor S1 (characteristics of literature) as more likely to induce literature anxiety than the situational factors S4 (tutorial groups) or S5 (time).

This finding is exemplified in the case of a participant called Fiona (pseudonym). Fiona is an amiable woman who was already qualified as a teacher prior to entering the program. She expressed no qualms about following the course and demonstrated a keen and positive outlook towards learning Literature in English. However, Fiona did express qualms about offering individual interpretations as, according to her, the lecturers did not always readily accept the students' interpretations and some lecturers did not always justify the students in why their answers were not accepted.

"Literature is very subjective. Sometimes ... even when we are in class, we are giving our ideas, we feel that what we analyzed and what we think we derived from the text is okay, but when we put forth to our lecturers, they say, "No, it's actually this way." So, you feel that you're wrong. Why is it not accepted, you know?"

There was also a significant difference between S2 (course structure, mean rank 5.62) and these factors:

- S3 (lecturer, mean rank 7.31), with $p=0.032 *$;

- S5 (time, mean rank 3.64), with $p=0.005^{* *}$;

- S6 (comprehension, mean rank 7.05), with $p=0.048^{*}$; and

- $\quad$ E2 (age, mean rank 3.93), with $p=0.01^{* *}$. 
This indicates that the participants were more likely to attribute the situational factor S3 (lecturer) as cause for provoking literature anxiety than the situational factor S2 (course structure). In the case of the situational factors S2 (course structure) and S5 (time), the participants were more likely to view S2 (course structure) as causing them literature anxiety than S5 (time). Between the situational factors S2 (course structure) and S6 (comprehension), participants were more likely to view S6 (comprehension) as provoking literature anxiety than S2 (course structure). Lastly, between the situational factors S2 (course structure) and E2 (age), the participants were more likely to view S2 (course structure) as inducing literature anxiety in them.

There was also a significant difference between S3 (lecturer, mean rank 7.31) and the following factors:

- S4 (tutorial groups, mean rank 4.10), with $p=0.003^{* *}$;

- S5 (time, mean rank 3.64), with $p=0.001^{* * *}$;

- D1 (attitude, mean rank 5.79), with $p=0.03^{*}$;

- E1 (educational background), mean rank 4.05, with $p=0.002^{* *}$; and

- $\quad$ E2 (age, mean rank 3.93), with $p=0.000^{* * *}$.

This indicates that the participants were more likely to attribute the situational factor S3 (lecturer) as causing them literature anxiety than the situational factor S4 (tutorial groups). In the case of the situational factors S3 (lecturer) and S5 (time), the participants were more likely to view S3 (lecturer) as causing them literature anxiety than S5 (time). Between S3 (lecturer) and D1 (attitude), E1 (educational background), and E2 (age), respectively, the participants were more inclined to report S3 (lecturer) as inducing literature anxiety for them.

The following examples of participant experiences depict what factors they were more likely to mention as inducing literature anxiety for them. Fiona (medium-anxiety group) was already a teacher when she entered the program. She questioned the teaching actions of some of the lecturers concerned whom she felt were invoking the experience of literature anxiety in them. She was also aware of the traits and teaching styles of these lecturers that made her anxious during her study of Literature in English.

Generally, Marge (pseudonym) (low-anxiety group) was very confident and positive about her Literature in English learning experiences. She also professed to having little anxiety about Literature in English. Armed with a keen and astute sense of learning, she demonstrated a strong liking for Literature in English. However, Marge did mention what made her anxious:

"We were very indignant about what $\operatorname{Dr} Y$ (pseudonym) did. Dr Y said that Dr $Y$ had to deduct marks because we plagiarized. But we didn't. We should have got an $A$, but Dr Y gave us a B+. There were only two $B+$ 's and the rest were $B$-'s and $C+$ 's. That really pulled down most people's grades. So, I went to explain to Dr Y that we did not plagiarize. Dr Y said most people plagiarized. But, of course, in the end, it's Dr Y's opinion and there is nothing you can do in that sense, because Dr Y is the marker. So, this is what makes me anxious." 
Marge recalled the abovementioned incident when she approached the lecturer concerned to seek clarification over the issue. The power relation between lecturer and student here made it difficult for Marge to communicate effectively with the lecturer. Thus, she acknowledged that this induced anxiety for her as the lecturer can impact the student's learning of Literature in English. It was situational factor S3 (lecturer) that induced literature anxiety for Marge.

Fiona's (medium-anxiety group) experiences, in a way, reflect those of Marge in the sense that the lecturer-student relation in class induced literature anxiety for Fiona.

"Sometimes some lecturers cannot take it when we throw back a question at them, you know, like me being a senior student in here, sometimes the lecturer's age and mine would be around the same, you know. This is what they want, and they are fixed with that kind of idea and that makes me anxious."

This behavior by the lecturer would affect Fiona's Literature in English learning in class, suggesting that passivity and acceptance seem to be more acceptable behavior in class, at least where certain lecturers were concerned.

There was also a significant difference between S4 (tutorial groups, mean rank 4.10) and:

- S6 (comprehension, mean rank 7.05), with $p=0.001^{* * *}$;

- D1 (ability, mean rank 5.79), with $p=0.031^{*}$; and

- $\quad$ D2 (attitude, mean rank 6.50), with $p=0.019^{*}$.

This suggests that the participants were more inclined to perceive the situational factor S6 (comprehension) as inducing literature anxiety for them than the situational factor S4 (tutorial groups). The participants were also more inclined to cite factors D1 (attitude) and D2 (ability) than S4 (tutorial groups) as provoking literature anxiety for them.

There was also a significant difference between S5 (time, mean rank 3.64) and:

- S6 (comprehension, mean rank 7.05), with $p=0.001^{* * *}$;

- D1 (attitude, mean rank 5.79), with $p=0.005^{* *}$; and

- D2 (ability, mean rank 6.50), with $p=0.003^{* *}$.

This suggests that the participants were more inclined to perceive the situational factor S6 (comprehension) as inducing literature anxiety for them than the situational factor S5 (time). They were also more inclined to perceive the dispositional factors D1 (attitude) and D2 (ability) rather than the situational factor S5 (time) as inducing literature anxiety.

There was also a significant difference between S6 (comprehension, mean rank 7.05) and:

- E1 (educational background, mean rank 4.05), with $p=0.001^{* * *}$; and

- E2 (age, mean rank 3.93), with $p=0.001^{\star * *}$. 
This suggests that the participants were more inclined to perceive the situational factor S6 (comprehension) as inducing literature anxiety for them than the environmental factors E1 (educational background) and E2 (age).

Aileen (pseudonym), a participant in the high-anxiety group, expressed her literature anxiety when she could not understand the literary material that she was studying:

$$
\text { "I feel anxious when I don't understand." }
$$

There was also a significant difference $\left(p=0.007^{* *}\right.$ ) between D1 (attitude, mean rank 5.79) and E2 (age, mean rank 3.93). This suggests that the participants were more inclined to perceive the dispositional factor D1 (attitude) as inducing literature anxiety for them than the environmental factor E2 (age).

Hetty (pseudonym), a participant in the medium-anxiety group, expressed her feeling that literature is difficult.

"I have never been anxious about other matters. For the first time, I feel only literature makes me anxious; literature is difficult."

Lastly, there was also a significant difference between D2 (ability, mean rank 6.50) and:

* E1 (educational background, mean rank 4.05); with $p=0.004^{* *}$; and

* E2 (age, mean rank 3.93), with $p=0.004^{* *}$.

This suggests that the participants were more inclined to perceive the dispositional factor D2 (ability) as inducing literature anxiety for them than the environmental factors E1 (educational background) and E2 (age).

Sally (pseudonym) (low-anxiety group) explained that even though she perceived herself to have invested time and effort in her literary tasks, she was unable to perform well. Sally seemed to question her ability to perform well in Literature in English and this induced literature anxiety in her. She was already a qualified teacher when she became a student. She enjoyed studying Literature in English very much and professed a desire to learn as much as she could about the course. Unfortunately, even though Sally's LITAS score placed her within the low-anxiety group, what hampered her enjoyment of studying Literature in English and caused her to feel anxious were her grades for Literature in English, which in a way resulted from the lecturer/lecturers concerned who assessed her. Sally felt that she had invested a lot of time, energy, and effort in her literature study, but her efforts did not reflect her achievement for Literature in English.

Don't know, I get anxious, but I say what can I do? I've put in and sacrificed a lot.

In summation, the participants were more likely to perceive the situational factor S1 (characteristics of literature) as inducing literature anxiety for them as compared to the situational factors S4 (tutorial groups) and S5 (time). Between the situational factors S2 (course structure) and S3 (lecturer), the participants were more inclined to view S3 (lecturer) as inducing literature anxiety. Between the situational factors S2 (course structure) and S5 (time), the participants were 
more likely to view the situational factor S2 (course structure) as invoking literature anxiety. In comparison to S2 (course structure), the participants were more inclined to view the situational factor S6 (comprehension) as inducing literature anxiety than S2 (course structure). When comparing the situational factor S3 (lecturer) with the situational factor S5 (time), dispositional factor D1 (attitude), and environmental factors E1 (educational background) and E2 (age), the participants were more inclined to perceive S3 (lecturer) as inducing literature anxiety. Thus, the S3 (lecturer) factor appeared to be more likely perceived by the participants to induce literature anxiety than the other factors of S5 (time), D1 (attitude), E1 (educational background) and E2 (age).

Furthermore, the situational factor S4 (tutorial groups) was less likely to be perceived by the participants as anxiety-inducing factor in comparison to the dispositional factors D1 (attitude) and D2 (ability) and the situational factor S6 (comprehension). The situational factor S6 (comprehension) was more likely to be perceived as inducing literature anxiety than the situational factor S5 (time) and the dispositional factors D1 (attitude) and D2 (ability) as well as the environmental factors E1 (educational background) and E2 (age). Thus, S6 (comprehension) appeared more likely to be perceived to induce literature anxiety than S5 (time), D1 (attitude), D2 (ability), E1 (educational background), and E2 (age). The situational factors S3 (lecturer) and S6 (comprehension) thus appear to stand out among the other factors as being more likely to induce literature anxiety among students.

\section{Conclusion}

The findings of this study revealed that the more competent the participants were in Literature in English, the less anxious they seemed to be. Through the participants' shared experiences, they confirmed that literature anxiety can be debilitating and, if unchecked, it could result in detrimentally affecting their results for Literature in English. Recognition of literature anxiety is essential so that evaluation of the potential sources of the anxiety can be studied and ways to alleviate this anxiety can be sought. Anxiety-provoking factors and the effective strategies employed by the respective students need to be tapped into considerably to assist students to reduce their literature anxiety levels to achieve effective Literature in English learning. It is imperative that research continues to further explore situations that provoke literature anxiety. Although this study explored a particular group of students in a specific setting, replications of this study with Literature in English learners of varying levels of competency in the subject and from different learning contexts is needed to understand how effectively the study's findings may be generalized to other Literature in English students elsewhere. The implications are that educators should teach more attentively and be sensitized to learners' possible experiences of anxiety.

\section{Funding}

This publication was supported by the authors' university grant (1001/PHUMANITI/8016022). 


\section{References}

Aydin, S. (2016). A qualitative research on foreign language teaching anxiety. The Qualitative Report, 21(4), 629-642. https:/ / doi.org/10.57643/2160-3175/2016.2232

Baloğlu, M. (2003). Individual differences in statistics anxiety among college students. Personality and Individual Differences, 34(5), 855-865. https://doi.org/10.1016/s0191-8869(02)00076-4

Bryman, A. (2004). Social research methods (2nd ed.). Oxford University Press.

Cheng, Y. S. (2004). A measure of second language writing anxiety: Scale development and preliminary validation. Journal of Second Language Writing, 13(4), 313-335. https://doi.org/10.1016/j.jslw.2004.07.001

Cheng, Y. S. (2017). Development and preliminary validation of four brief measures of L2 language-skill-specific anxiety. System, 68, 15-25. https:// doi.org/10.1016/j.system.2017.06.009

Corbin, J., \& Strauss, A. (2008). Basics of qualitative research: Techniques and procedures for developing Grounded Theory (3rd ed.). Sage.

Deieso, D., \& Fraser, B. J. (2018). Learning environment, attitudes and anxiety across the transition from primary to secondary school mathematics. Learning Environment Research, 22, 133-152. https:// doi.org/10.1007/s10984-018-9261-5

Ellis, R. (1994). The study of second language acquisition. Oxford University Press.

Endler, N. S., \& Kocovski, N. L. (2001). State and trait anxiety revisited. Journal of Anxiety Disorders, 15(3), 231-245. https://doi.org/10.1016/s0887-6185(01)00060-3

Horowitz, E. K., Horowitz, M. B., \& Cope, J. (1986). Foreign language classroom anxiety. The Modern Language Journal, 70(2), 125-132. https:// doi.org/10.2307/327317

Huang, H.-T. D. (2018). Modeling the relationships between anxieties and performance in second/foreign language speaking assessment. Learning and Individual Differences, 63, 44-56. https://doi.org/10.1016/j.lindif.2018.03.002

Jiang, Y., \& Dewaele, J.-M. (2020). The predictive power of sociobiographical and language variables on foreign language anxiety of Chinese university students. System, 89, 102207. https:// doi.org/10.1016/j.system.2020.102207

Lou, N. M., \& Noels, K. A. (2020). Breaking the vicious cycle of language anxiety: Growth language mindsets improve lower-competence ESL students' intercultural interactions. Contemporary Educational Psychology, 61, 1-17. https:// doi.org/10.1016/j.cedpsych.2020.101847

Luttenberger, S., Wimmer, S., \& Paechter, M. (2018). Spotlight on math anxiety. Psychology https://doi.org/10.2147/prbm.s141421

Macintyre, P. D. (1995). How does anxiety affect second language learning? A reply to Sparks and Ganschow. The Modern Language Journal, 79(1), 90-99. https://doi.org/10.1111/j.1540-4781.1995.tb05418.x

Martinussen, L. J., Šaltytė Benth, J., Almdahl, I. S., Borza, T., Selbæk, G., Mcpherson, B., \& Korsnes, M. S. (2019). The effect of anxiety on cognition in older adult inpatients with depression: Results from a multicenter observational study. Heliyon, 5(8), e02235. https://doi.org/10.1016/j.heliyon.2019.e02235

Neuman, L. A. (2000). Social research methods: Qualitative and quantitative approaches (4th ed.). Allyn and Bacon.

Ohata, K. (2005). Language anxiety from the teacher's perspective: Interviews with seven experienced ESL/EFL teachers. Journal of Language and Learning, 3(1), 133-155.

Oppenheim, A. N. (1992). Questionnaire design, interviewing and attitude measurement (New ed.). Continuum.

Putwain, D. W., Connors, L., \& Symes, W. (2010). Do cognitive distortions mediate the 
test anxiety-examination performance relationship? Educational Psychology, 30(1), 11-26. https:// doi.org/10.1080/01443410903328866

Reardon, T., Harvey, K., Young, B., O'Brien, D., \& Creswell, C. (2018). Barriers and facilitators to parents seeking and accessing professional support for anxiety disorders in children: Qualitative interview study. European Child $\mathcal{E}$ Adolescent Psychiatry, 27(8), 1023-1031. https:// doi.org/10.1007/s00787-018-1107-2

Salza, A., Giusti, L., Ussorio, D., Casacchia, M., \& Roncone, R. (2020). Cognitive behavioral therapy (CBT) anxiety management and reasoning bias modification in young adults with anxiety disorders: A real-world study of a therapist-assisted computerized (TACCBT) program vs. "person-to-person" group CBT. Internet Interventions, 19, 100305. https:// doi.org/10.1016/j.invent.2020.100305

Spielberger, C. D., \& Vagg, P. R. (Eds.). (1995). Test anxiety: Theory, assessment and treatment. Taylor \& Francis.

Zaccoletti, S., Altoè, G., \& Mason, L. (2020) Enjoyment, anxiety and boredom, and their control-value antecedents as predictors of reading comprehension. Learning and Individual Differences, 79, 101869. https:/ / doi.org/10.1016/j.lindif.2020.101869 